\title{
Wall accumulation of self-propelled spheres
}

\author{
Jens Elgeti And Gerhard Gompper \\ Theoretical Soft Matter and Biophysics, Institute of Complex Systems and Institute for Advanced Simulation, \\ Forschungszentrum Jülich, Germany
}

PACS 82.70.-y - Disperse systems; complex fluids

PACS 45.50.- $\mathrm{j}$ - Dynamics and kinematics of a particle and a system of particles

PACS 05.40.-a - Fluctuation phenomena, random processes, noise, and Brownian motion

\begin{abstract}
The dynamics of a self-propelled Brownian sphere confined between two planar hard walls is investigated by computer simulations and analytic solutions of the corresponding FokkerPlanck equation. It is shown that an accumulation of self-propelled particles, often linked to the hydrodynamic dipole interaction, can be already obtained from the combination of Brownian motion and self-propulsion. The surface excess is calculated as a function of particle velocity, wall separation, and translational and rotational diffusion coefficients. In limits of narrow channels or small propulsion velocities, analytical solutions and numerical results are in excellent agreement.
\end{abstract}

Introduction. - Many uni- and multi-cellular organisms employ self-propulsion to find food, to transport a cargo, or to evade unfavorable environmental conditions. Well-known examples are bacteria [1], sperm, and algae [2]. The study of the processes and strategies employed by such biological microswimmers to move through a viscous fluid is not only important to understand biological systems and affect their behavior, but also to understand the behavior of active systems in general $[3,4]$ and to construct efficient biomimetic microswimmers [5]. Furthermore, much progress has been made recently to design artificial nano- and microswimmers, which use propulsion principles not known from biological systems $[3,6]$.

Microswimmers hardly ever swim alone in an unbounded fluid - they rather interact with other swimmers, and with walls and obstacles. In these interactions, three main contributions can be distinguished: (i) volume exclusion, which makes the swimmers avoid each other at short distances, (ii) hydrodynamic interactions, which lead to alignment and attraction or repulsion depending on the type of propulsion mechanism (pusher, puller, or neutral swimmer), and (iii) thermal or intrinsic noise, which implies rotational diffusion of the swimming direction. The relative importance of propulsion and noise can be characterized by the Peclet number $P e_{0}=v_{0} /\left(D_{r} R_{0}\right) \sim$ $v_{0} \eta R_{0}^{2} /\left(k_{B} T\right)$, where $v_{0}$ is the swimming velocity, $D_{r}$ the rotational diffusion constant, $\eta$ the fluid viscosity, $R_{0}$ a characteristic swimmer size and $k_{B} T$ the thermal energy. For fast swimmers like sperm, Peclet numbers are of the order $10^{4}-10^{5}$ (neglecting the effect of biological noise on rotational diffusion), while for slow swimmers, Peclet numbers are in the range $P e_{0} \simeq 5-200$ for diffusiophoretic Janus spheres with radii between $0.25-5 \mu \mathrm{m}$, respectively $[7,8]$, and $P e_{0}=120$ for E. coli [9]. It has been shown for Chlamydomonas reinhardtii that the noise inherent to the biological machines is very important: it reduces the effective Peclet number for these algae from $\mathrm{Pe}=\mathcal{O}\left(10^{4}\right)$ to $P e=50$ [9]. It is important to note that for $P e=\mathcal{O}\left(10^{2}\right)$, rotational diffusion is strong enough to significantly affect the trajectories of interacting microswimmers $[9,10]$. For dipole swimmers, the strength of the hydrodynamic far-field interactions is proportional to the swimming velocity, and therefore directly related to the Peclet number; furthermore, for neutral swimmers, the hydrodynamic interaction is very weak $[3,4]$.

Sperm [11] and E. coli [12] have been found to strongly adhere to the walls of an observation chamber. Different mechanisms have been proposed to explain and predict this behavior. For high-Pe swimmers, hydrodynamic interactions play an essential role. In particular, force-dipole and source-dipole contributions have been studied in detail $[13,14]$. For low-Pe swimmers, other mechanisms of surface accumulation may dominate, such as the alignment of rod-like swimmers parallel to a wall $[15,16]$, or crowding [17]. Indeed, experimental evidence suggests that in wall accumulation of E. coli, hydrodynamic interactions play a minor role [9]. In fact, for a microswimmer close to a wall, it is most likely the combination of excluded volume 

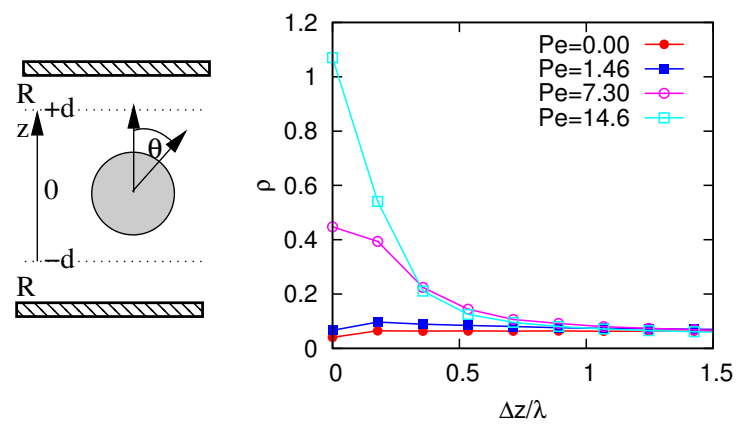

Fig. 1: (Left) A self-propelled Brownian sphere of radius $R$ is confined between two solid walls at $z= \pm(d+R)$. The orientation of the propulsion direction relative to the $\mathrm{z}$ axis, is denoted by $\theta$. (Right) Probability density $\rho(\Delta z)$ to find a particle at a distance $\Delta z$ from the wall. At zero Peclet number $P e=v_{0} / \sqrt{D_{r} D}$, the probability density is uniform beyond the short range of the repulsive wall. With increasing Peclet number particles accumulate near the wall. Results are shown for a system with wall separation $d / \lambda=15.6$. The curves correspond to a surface excess of $s=0,0.05,0.24$, and 0.38 with increasing Peclet number, respectively.

and full hydrodynamic (beyond the dipole approximation) interactions which is responsible for wall adhesion [18].

Another interesting class of self-propelled particles are cells and microorganisms - such as keratocytes [19] or Myxococus xanthus [20] - which move on a surface by crawling or sliding, or cancer cell moving in a threedimensional gel matrix [21]. In this kind of motion, hydrodynamics should be irrelevant. Such self-propelled particles can also hit obstacles or walls on their way and may, or may not, adhere to them.

In the studies of self-propelled rods near surfaces, it was assumed that the elongated shape of the rod is essential for the accumulation $[15,16]$. We want to consider here an even simpler system of a self-propelled Brownian sphere. Surprisingly, we find that a self-propelled sphere shows even stronger accumulation at a wall than a self-propelled rod. Furthermore, the simplicity of a spherical particle allows for an analytical solution of the Fokker-Planck equation in limits of small wall separations or small Peclet numbers. The analytic results agree well with the simulation results in the appropriate limits, and help to understand the origin of surface accumulation of microswimmers in the absence of hydrodynamic interactions.

Model and Simulation Technique. - The method of Multi-Particle Collision dynamics (MPC) is well established for studying microswimmers [22] and self-propelled particles [15]. An advantage of MPC is a natural inclusion of thermal fluctuations. Furthermore the hydrodynamic interactions can be turned on and off, without effect on thermal properties. We employ here a variant of MPC without hydrodynamic interactions, called random MPC or Brownian MPC, which is equivalent to Brownian Dy- namics simulations [23]. The fluid is modeled only by its effect on suspended particles, which are constructed from a collection of point-like monomers. After a propagation time $h$, in which monomers move according to Newton's equation of motion, the monomer particles collide with fluid particles. In random $\mathrm{MPC}$, random thermal velocities are assigned to the fluid particles in each collision step. Thus, each monomer interacts with $\rho_{s}=10$ fluid particles of mass $m$ and thermal velocities $\mathbf{v}=0.5 \sqrt{k_{B} T / m} \mathbf{N}(0,1)$ (with $\mathbf{N}(0,1)$ a vector whose components are standard normal distributed). The collision step then consists of a random rotation of the relative velocity, as in standard MPC $[24,25]$. This allows us to study the effects of noise and self-propulsion, without the interference of hydrodynamics.

The sphere of radius $l_{0}$ is represented by $N=12$ point particles of monomer mass $M=5 \mathrm{~m}$ in a icosahedral arrangement. These monomers are connected to their neighbors by strong harmonic potentials $U_{b}=0.5 K_{b}\left(|\Delta r|-l_{b}\right)^{2}$ with spring constant $K=2 \cdot 10^{5} k_{B} T / l_{0}^{2}$ and bond length $l_{b}=1.05146 l_{0}$, plus an additional particle at the center, bonded to all other particles by a similar potential with spring constants $K_{c}=K_{b} / 10$ and bond length $l_{c}=l_{0}$. This central particle is repelled from the wall by a shifted and truncated Lennard Jones potential, with $\epsilon=k_{B} T$ and cutoff $0.25 l_{0}$. A constant driving force $\mathbf{F}_{i}=F\left(\mathbf{r}_{1}-\mathbf{r}_{2}\right)$ is added to all particles of the colloid where $\mathbf{r}_{1 / 2}$ are the positions of two designated monomers on opposite sides of the colloid, which define the sphere orientation. For a swimmer in a hydrodynamic solvent, the location of the fluid reaction force is very important, as it greatly influences the swimmer interactions [14]. However, in Brownian dynamics there is no reaction force on the fluid, and therefore also no hydrodynamic interaction with the walls.

From simulations of freely diffusing spheres we extract the diffusion coefficient $D=6.6 \cdot 10^{-4} l_{0} \sqrt{k_{B} T / m}$ and rotational diffusion coefficient $D_{r}=2.1 \cdot 10^{-3} \sqrt{k_{B} T / m} / l_{0}$. The corresponding hydrodynamic radius of the colloid is thus $R=\sqrt{0.75 D / D_{r}}=0.49 l_{0}$. The actual radius of the sphere has however no influence on the dynamics of the sphere, besides this ratio. Indeed it turns out in the analytic calculations below that the natural length scale in the problem is the ratio of translational and rotational diffusion coefficients. We thus define a characteristic length scale

$$
\lambda=\sqrt{D / D_{r}}
$$

In our simulation model, $\lambda \approx 0.566 l_{0}$. Without hydrodynamics, the velocity is directly proportional to the driving force $F$, with a mobility $D / k_{B} T$, i.e. $v_{0}(F)=D F / k_{B} T$. In combination with the length scale $\lambda$, we define the Peclet number

$$
P e=\frac{v_{0} \lambda}{D}=\frac{v_{0}}{\sqrt{D_{r} D}} .
$$

Simulation Results. - Self-propulsion leads to accumulation of particles near the wall. The simulation results shown in fig. 1 demonstrate that the (normalized) 


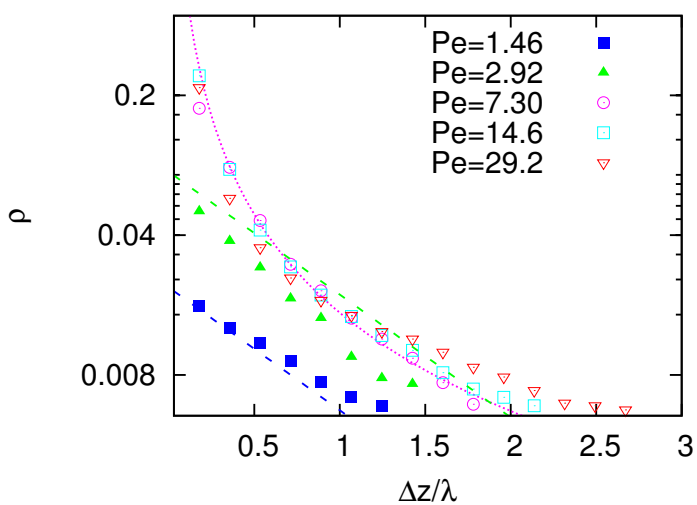

Fig. 2: Probability density increment $\left(\rho(z)-\rho_{b}\right)$ to find a particle at a distance $\Delta z$ from the wall. Simulation data are shown for a system with wall separation $d / \lambda=15.6$. For small Peclet number $P e<2$, the probability density is exponential, and well reproduced by an analytic expression (15) (dashed lines). At higher Peclet numbers, the probability density increment is well described by a power law with an exponent of approximately 1.6 (dotted line).

probability density $\rho(\Delta z)$ to find a particle at a distance $\Delta z$ from the wall is strongly peaked close to the wall for $P e \gtrsim 5$. Contrary to our expectation, the effect is even stronger than for rods, and does not saturate with increasing Peclet number.

A natural expectation for the spatial dependence of $\rho(\Delta z)$ is an exponential decay. This is indeed seen in the simulation results for small Peclet numbers (see fig. 2). However, for larger Peclet numbers with $P e>7$, the decay of probability density away from the wall seems to be better described by a power law. On the other hand, the analytic calculations presented below suggest a multiexponential form of the distribution. Because of the short range over which the probability density decays, it is not possible to distinguish between the two hypotheses on the basis of our simulations.

To quantify the dependence of the wall accumulation on Peclet number and wall separation, we introduce the surface excess $s$, which is defined as

$$
s=1-\int_{-\alpha d}^{\alpha d} \rho(z) d z / \int_{-\alpha d}^{\alpha d} \rho_{0}(z) d z
$$

where $\rho_{0}(z)$ is the probability density for the unpropelled sphere. Here, $\alpha=1 / 2$ is chosen such as to restrict the integral over the bulk region of the density profile. For hard walls, eq. (3) is equivalent to $s=\int_{-d}^{d}\left(\rho-\rho_{b}\right) d z$ with bulk density $\rho_{b}$. The main advantage of eq. (3) is a simpler numerical implementation and lower sensitivity to noise. $s$ vanishes for a homogeneous distribution, is negative when the particle is repelled from the wall, and approaches unity for complete adhesion.

Figure 3 shows the surface excess as a function of the Peclet number for different channel widths. Note that $s$

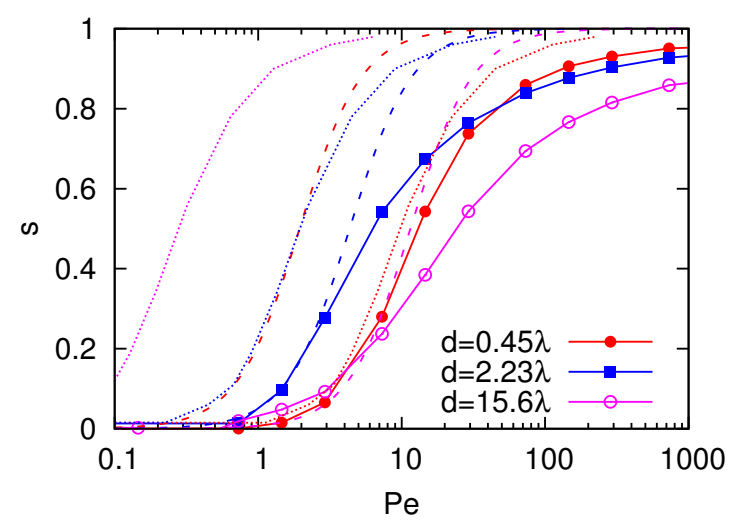

Fig. 3: Surface excess $s$ as a function of Peclet number Pe, for various wall separations $d$ as indicated. Results from the analytic calculation for very thin channels (dotted lines) match well the simulations for very thin channels, but fail for wider channels. The approximation for small Peclet numbers (dashed lines) works well for large wall separations and small Peclet number. It overestimates the surface excess for large $P e$. All analytic expression have no adjustable parameters.

does not saturate to a value $s_{\max }<1$ as was observed for self-propelled rods [15], but approaches unity for large $P e$ (complete adhesion).

The dependence of $s$ on channel width $d$, shown in fig. 4, comes somewhat at a surprise. Initially the the surface excess increases rapidly with $d$, up to a maximum at $d / \lambda \approx 2$, and then slowly decreases again. However, these behaviors can be well understood from solutions of certain limits of the Fokker Planck equation, as explained in detail below.

Fokker-Planck description. - To understand the underlying principles of why self-propelled spheres adhere to boundaries, it is useful to study the corresponding continuum theory. We describe the system in terms of the probability density $\rho(\mathbf{r}, \mathbf{n}, t)$ to find the sphere at position $\mathbf{r}$ with orientation $\mathbf{n}$ at time $t$,

$$
\partial_{t} \rho(\mathbf{r}, \mathbf{n}, t)=\left[D_{r} \nabla_{n}^{2}-v_{0} \mathbf{n} \cdot \nabla_{r}+D \nabla_{r}^{2}\right] \rho(\mathbf{r}, \mathbf{n}, t)
$$

Symmetry allows us to greatly simplify phase space to effectively two dimensions, the distance $z$ from the central plane, and the orientation angle $\theta$ (see fig. 1). The FokkerPlanck equation then reads

$$
\begin{aligned}
\partial_{t} \rho(z, \theta, t) & =D_{r} \frac{1}{\sin (\theta)} \partial_{\theta}\left[\sin (\theta) \partial_{\theta} \rho(z, \theta, t)\right] \\
& -v_{0} \cos (\theta) \partial_{z} \rho(z, \theta, t)+D \partial_{z}^{2} \rho(z, \theta, t)
\end{aligned}
$$

where $\theta=0$ corresponds to particles oriented in the positive $z$-direction.

The principle origin of surface accumulation for selfpropelled spheres can be identified from eq. (5). In the case of a self-propelled sphere, the rotational diffusion is independent of position and orientation, so that the average orientation is isotropic. Thus all particles with a 


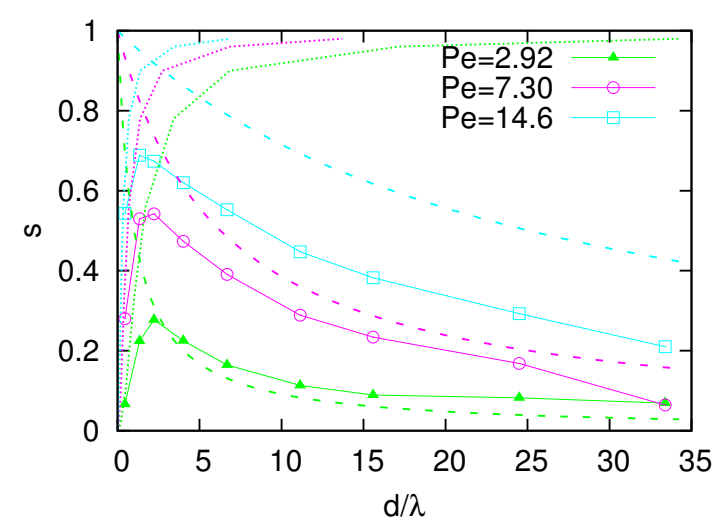

Fig. 4: Surface excess $s$ as a function of wall separation $d$, for three different Peclet numbers, as indicated. $s$ first increases sharply with increasing wall separation, but then slowly vanishes as the channel gets wider. The approximation for small Peclet numbers (dashed lines) fails to quantitatively predict the surface excess (because $P e>2$ ), but captures the correct trends for wider channels. The thin-channel approximation (dotted lines) shows the increase of $s$ with increasing channel width. All analytic expression have no adjustable parameters.

positive orientation will move quickly to the top wall, and all particles with negative orientation to the bottom wall. The bulk is thus depleted of particles, and particles at the boundaries are homogeneously distributed over all angles facing towards the wall. Therefore, the driving force does not order the particle orientations, it just sorts them in different regions like a gravitational force $\propto v_{0} \cos (\theta)$. Noise than acts in the form of translational diffusion, yielding an exponential distribution as for settling colloidal spheres.

Thin films. It is useful to define two Peclet numbers $P e_{r}=v_{0} / D_{r} d$ and $P e_{d}=v_{0} d / D$ (note that $P e_{d}=$ $\operatorname{Ped} / \lambda)$. In steady state, and after rescaling lengths by the effective channel width $d$ (the width of the channel that the center of mass of the sphere can explore), eq. (5) reads

$$
\cos (\theta) \partial_{z} \rho=P e_{r}^{-1} \sin (\theta) \partial_{\theta} \sin (\theta) \partial_{\theta} \rho+P e_{d}^{-1} \partial_{z}^{2} \rho
$$

To solve the eq. (6), we begin with a "thin-channel" approximation. Because for a no-slip sphere $D_{r}=$ $k_{B} T /\left(8 \pi \eta R^{3}\right), D=k_{B} T /(6 \pi \eta R)$, and

$$
\frac{P e_{r}}{P e_{d}}=\frac{D}{d^{2} D_{r}}=\frac{4}{3}\left(\frac{R}{d}\right)^{2},
$$

rotational diffusion is "slow" in the limit of thin channels with $R / d \ll 1$, and $P e_{r}^{-1}$ can be assumed to be negligible. (Note that $d$ can indeed be smaller than $R$, because $2 d$ is the width of the channel that the center of the sphere can explore (see fig. 1); the true channel width is $2 d+$ $2 R$.) Even though this limit is only strictly valid for very thin channels, it gives a good indication about the origin of the surface accumulation, especially for higher Peclet numbers.
The boundary conditions are zero flux $J$ at both walls at $\pm d$ and overall isotropy,

$$
\begin{aligned}
0=J( \pm 1) & =\rho( \pm 1, \theta) \cos (\theta)-P e_{d}^{-1} \partial_{z} \rho( \pm 1, \theta) \\
\int_{-1}^{1} \rho(\theta, z) d z & =2 \rho_{0} / \pi
\end{aligned}
$$

The limit $P e_{r} \rightarrow \infty$ greatly simplifies eq. (6). After $z$-integration, eq. (6) becomes $P e_{d} \cos (\theta) \rho=\partial_{z} \rho$. A second $z$-integration and application of the boundary conditions then yields

$$
\rho(z, \theta)=\frac{P e_{d} \rho_{0} \cos (\theta)}{\pi \sinh \left(P e_{d} \cos (\theta)\right)} e^{P e_{d} \cos (\theta) z}
$$

The surface excess $s$ is evaluated numerically using eq. (3), and is shown for different channel widths in fig. 3. It agrees well with the simulation results for thin channels, but obviously fails for wider channels. Note that the surface excess is symmetric in $P e_{d}$. In a more detailed comparison (see fig. 5) we see that the agreement is even quantitative for the full probability density as a function of position and orientation for a very thin channel. At high $P e_{d}$, particles completely sort to the wall they are pointing to. With a sharp exponential decay (decay length $\left.\propto 1 /\left(P e_{d} \cos (\theta)\right)\right)$, the probability density drops to zero towards the center-line. Only particles oriented almost parallel to the surface can be found in the center. Indeed, eq. (9) predicts a uniform distribution of particles oriented exactly parallel to the surface. As the Peclet number decreases, the decay length increases and eventually exceeds the system size. Thus for smaller Peclet numbers, particles of all orientations can be found everywhere, with just a slight increase for particles pointing towards the surface, and a small decrease for particles pointing away. However even for $P e_{d} \approx 1$ particles in the center are not uniformly distributed. The boundary condition of the next section (see eq. (13)) thus does not apply in this case. For wider channels, the approximation fails as rotational diffusion becomes more important.

Small Peclet numbers. The onset of accumulation of particles at a wall for small Peclet numbers and the effect of wider channels can best be understood by a different approach. We consider now a semi-infinite system with a wall at $z=0$, and an isotropic bulk at infinity. We decompose the density in spherical harmonics,

$$
\rho(z, \theta, t)=\sum_{l=0}^{\infty} \tilde{\rho}_{l}(z, t) Y_{l}^{0}(\theta)
$$

where $Y_{l}^{0}(\theta)=P_{l}(\cos (\theta))$ are the spherical harmonics in "Schmidt normalization", and $P_{l}$ are the Legendre polynomials. Projecting on orthogonal modes and using properties of the Legendre polynomials, we rewrite eq. (5) as

$$
\begin{aligned}
\partial_{t} \tilde{\rho}_{l}(z, t) & =D \partial_{z}^{2} \tilde{\rho}_{l}(z, t)-D_{r} l(l+1) \tilde{\rho}_{l}(z, t) \\
& -\frac{l}{2 l-1} v_{0} \partial_{z} \tilde{\rho}_{l-1}(z, t)-\frac{l+1}{2 l+3} v_{0} \partial_{z} \tilde{\rho}_{l+1}(z, t)
\end{aligned}
$$



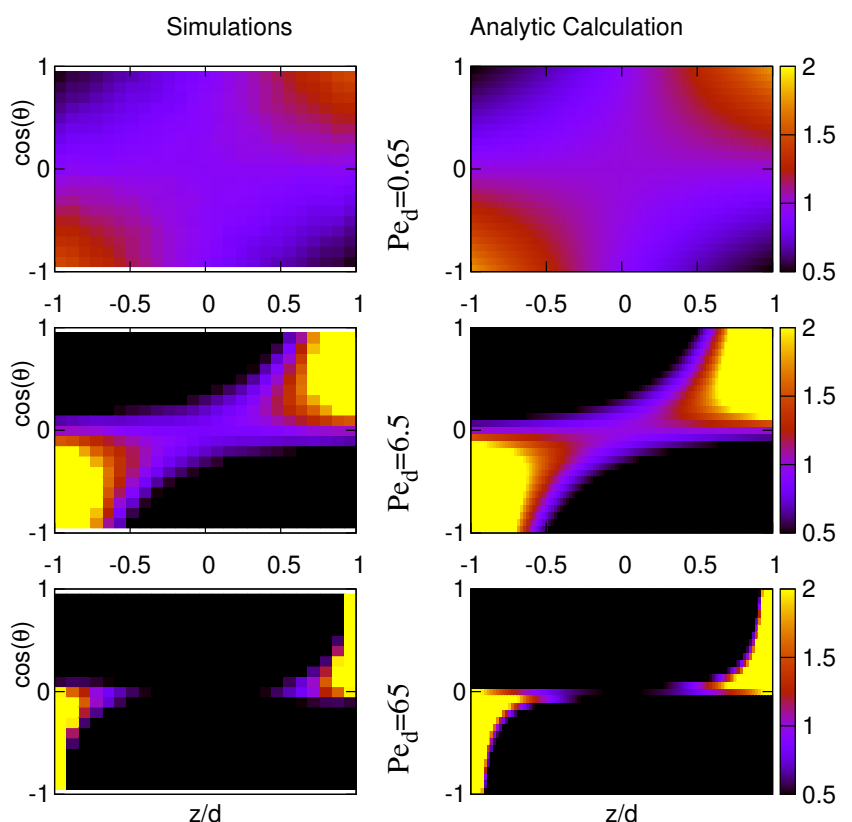

Fig. 5: Simulation (left) and theory (right) results for the relative probability density $\left(\rho / \rho_{0}\right)$ to find a particle in a thin channel of width $d=0.44 \lambda$. For very thin channels, the agreement between simulation and analytic result (9) is excellent without any adjustable parameters.
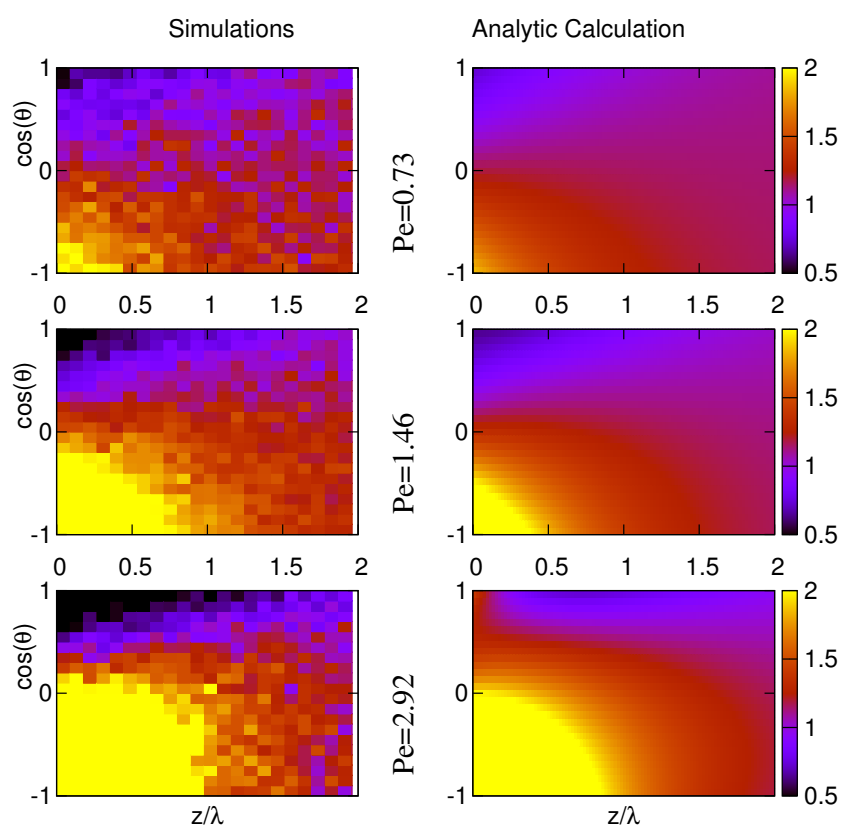

Fig. 6: Relative probability density $\left(\rho / \rho_{0}\right)$ to find a particle at a distance $z$ from the wall, for wider channels with $d=15.6 \lambda$. In the analytic expression (15), only the "bulk density" $\rho_{0}$ is adjustable, which is determined by averaging the histogram far from the walls. Excellent agreement is obtained for small Peclet numbers, but already at $P e \gtrsim 1$ quantitative differences become visible. In particular, at $P e=2.92$ second order theory predicts an accumulation even for particles pointing away from the surface, while simulations show a depletion of such particles.
As for the simulations, we employ the length scale $\lambda=$ $\sqrt{D / D_{r}}$ and the mixed Peclet number $P e=v_{0} / \sqrt{D D_{r}}$. In steady state, and after a change to coordinates $z^{\prime}=$ $z / \lambda$, we obtain

$$
\left(\partial_{z^{\prime}}^{2}-k_{l}^{2}\right) \tilde{\rho}_{l}=P e\left[\frac{l}{2 l-1} \partial_{z^{\prime}} \tilde{\rho}_{l-1}+\frac{l+1}{2 l+3} \partial_{z^{\prime}} \tilde{\rho}_{l+1}\right]
$$

with $k_{l}=\sqrt{l(l+1)}$. For the boundary conditions, we now choose an isotropic system at $z \rightarrow \infty$ and a zero flux wall at $z=0$,

$$
\begin{aligned}
\tilde{\rho}_{l}(\infty) & =\delta(l, 0) \\
0=J(0) & =\rho(0, \theta) v_{0} \cos (\theta)-D \partial_{z} \rho(0, \theta)
\end{aligned}
$$

so that

$$
0=\partial_{z^{\prime}} \tilde{\rho}_{l}-P e\left[\frac{l}{2 l-1} \tilde{\rho}_{l-1}+\frac{l+1}{2 l+3} \tilde{\rho}_{l+1}\right]
$$

We solve these equations in orders of Peclet number, and finally arrive at

$$
\begin{aligned}
\frac{\rho(z, \theta)}{\rho_{0}} & =1-\frac{P e}{\sqrt{2}} \exp (-\sqrt{2} z / \lambda) Y_{1}^{0}(\theta) \\
& +\frac{P e^{2}}{6}[\sqrt{3} \exp (-\sqrt{6} z / \lambda)-\exp (-\sqrt{2} z / \lambda)] Y_{2}^{0}(\theta) \\
& +\frac{P e^{2}}{6} \exp (-\sqrt{2} z / \lambda)+\mathcal{O}\left(P e^{3}\right)
\end{aligned}
$$

For the relative surface excess, this implies

$$
\begin{aligned}
s_{r} & =\frac{1}{\rho_{0}} \int_{0}^{\infty}[\rho(z)-\rho(\infty)] d z \\
& =\int_{0}^{\infty}\left[\frac{P e^{2}}{6} \exp (-\sqrt{2} z / \lambda)+\mathcal{O}\left(P e^{3}\right)\right] d z \\
& =\frac{\lambda P e^{2}}{6 \sqrt{2}}+\mathcal{O}\left(P e^{4}\right)
\end{aligned}
$$

In the limit of decay lengths much larger than the channel width, this relative surface excess relates to the surface excess via $s=s_{r} /\left(s_{r}+d\right)$. (Note that the $P e^{3}$ term in the surface excess vanishes due to symmetry, and thus eq. (16) is $\mathcal{O}\left(P e^{4}\right)$.) We thus see that also for wide channels, selfpropulsion leads to accumulation at the surface. To first order in $P e$, only the average orientation of the particle at the surface changes, such that the probability of orientation towards the wall increases by the same amount as the probability of orientation away from the wall decreases, but not the density. To second order in $P e$, the density at the surface increases as $P e^{2}$. Indeed, the symmetry that particles with velocities $\pm v_{0}$ show the same accumulation, dictates that all odd powers of $v_{0}$ vanish.

The surface excess predicted by eq. (16) to second order in $P e$, also displayed in fig. 3, again shows astonishingly good agreement with the simulation results for wider channels. Because the spherical harmonics are orthogonal, the probability density to find a particle at position $z$ only depends on the $\theta$-independent terms in eq. (15). To second 
order, we thus have an exponential decay to the bulk density as a function of distance from the surface. This fits well our simulation results (see fig. 2). Terms of higher order in $P e$ should be exponentials as well. Thus, for larger Peclet number we expect a sum of exponentials, and not a power law as indicated by the simulations.

Looking in detail at the distribution $\rho(z, \theta)$, see fig. 6, we find very good agreement for $P e<2$, and still the correct trends for $\mathrm{Pe}>2$. However, for particles with an orientation pointing away from the surface $(z=0$, $\cos (\theta)=1)$, the theory still predicts an accumulation, while the simulation show a depletion of those particles. This deviation is most probably due to the omission of higher-order terms.

In combination with the thin-channel approximation, we can now also understand the form of the channel-width dependence in fig. 4. As the channel width $d$ increases, the thin channel approximation applies for $d / \lambda \lesssim 1$, and $s$ is predicted to increase in this regime through the increasing Peclet number $P e_{d}$. However, as $d / \lambda$ exceeds unity, rotational diffusion becomes important, and the thin channel approximation fails. Instead, we can employ the small Peclet-number approximation, which predicts that the surface excess decays as $\left(1+d / s_{r}\right)^{-1}$, where $s_{r} \sim \lambda P e^{2}$ is independent of $d$.

Summary \& Conclusions. - We have shown that simple self-advection Brownian dynamics of spherical particles is sufficient to generate strong surface adhesion. This mechanism of accumulation at planar walls (or obstacles) is very generic. For example, a sphere with hydrodynamic slip at its surface has a different value of $\lambda$ than a noslip sphere, but otherwise will show exactly the same wall accumulation behavior. In more complex systems, e.g. for many swimmers in a hydrodynamic environment, additional effects like hydrodynamic interactions $[18,26]$ or crowding [17] can help or hinder this accumulation, but the competition of propulsion and orientational diffusion needs to be taken into account in all descriptions. The hydrodynamic slowing down of rotational diffusion close to a wall [27], will further enhance the surface accumulation.

Based on a Fokker-Planck description, we are able to understand how surface accumulation arises from the sorting of oriented particles away or towards a wall. To first order in Peclet number, this leads to an preferred orientation of particles towards the wall, but does not affect the density. Only to second order in Peclet number does this orientation lead to accumulation at the wall. For small Peclet numbers, this increased probability density drops exponentially to the bulk density on length scales $\sqrt{D /\left(2 D_{r}\right)}$. At higher Peclet numbers, the sum of several exponential decays is difficult to distinguish from an apparent powerlaw behavior observed in the simulations.

An interesting conclusion can also be obtained by comparing spherical and rod-like microswimmers. In the case of rods, the surface accumulation remains incomplete (with surface excess $s<1$ ) even for large swimming veloc- ities, while $s=1$ for spheres in this limit. Thus, an elongated shape actually helps the microswimmer to escape from the surface (in the absence of hydrodynamic attraction), because it suppresses orientations of the swimmer towards the wall.

\section{REFERENCES}

[1] Berg H. C., E. coli in Motion (Springer, New York) 2004.

[2] Polin M., Tuval I., Drescher K., Gollub J. P. and Goldstein R. E., Science, 325 (2009) 487.

[3] Lauga E. and Powers T. R., Rep. Prog. Phys., 72 (2009) 096601.

[4] Ishikawa T., J. R. Soc. Interface, 6 (2009) 815.

[5] Dreyfus R., Baudry J., Roper M. L., Fermigier M., Stone H. A. and Bibette J., Nature, 437 (2005) 862.

[6] Ozin G. A., Manners I., Fournier-Bidoz S. and ArSENAUlt A., Adv. Mater., 17 (2005) 3011.

[7] Howse J. R., Jones R. A. L., Ryan A. J., Gough T., Vafabakhsh R. and Golestanian R., Phys. Rev. Lett. 99 (2007) 048102.

[8] Ebbens S., Tu M.-H., Howse J. R. and Golestanian R., Phys. Rev. E, 85 (2012) 020401.

[9] Drescher K., Dunkel J., Cisneros L. H., Ganguly S. and Goldstein R. E., Proc. Natl. Acad. Sci. USA, 108 (2011) 10940.

[10] Götze I. O. and Gompper G., Phys. Rev. E, 82 (2010) 041921.

[11] Rothschild, Nature, 198 (1963) 1221.

[12] Lauga E., Diluzio W. R., Whitesides G. M. and Stone H. A., Biophys. J., 90 (2006) 400.

[13] Berke A. P., Turner L., Berg H. C. and Lauga E., Phys. Rev. Lett., 101 (2008) 038102.

[14] Spagnolie S. E. and Lauga E., J. Fluid Mech., 700 (2012) 105.

[15] Elgeti J. and Gompper G., EPL, 85 (2009) 38002.

[16] Li G. and Tang J. X., Phys. Rev. Lett., 103 (2009) 078101.

[17] Wensink H. H. and Löwen H., Phys. Rev. E, 78 (2008) 031409

[18] Elgeti J., Kaupp U. B. and Gompper G., Biophys. J., 99 (2010) 1018.

[19] Verkhovsky A. B., Svitkina T. M. and Borisy G. G.,Curr. Biol., 9 (1999) 11.

[20] Peruani F., Starruss J., Jakovljevic V., SøgaardAndersen L., Deutsch A. and Bär M., Phys. Rev. Lett., 108 (2012) 098102.

[21] Poincloux R., Collin O., Lizárraga F., Romao M., Debray M., Piel M. and Chavrier P., Proc. Natl. Acad. Sci. USA, 108 (2011) 1943.

[22] Yang Y., Elgeti J. and Gompper G., Phys. Rev. E, 78 (2008) 061903.

[23] Ripoll M., Winkler R. G. and Gompper G., Eur. Phys. J. E, 23 (2007) 349.

[24] Kapral R., Adv. Chem. Phys., 140 (2008) 89.

[25] Gomprer G., Ihle T., Kroll D. M. and Winkler R. G., Adv. Polym. Sci., 221 (2009) 1.

[26] Li G. L., Tam L. K. and Tang J. X., Proc. Natl. Acad. Sci. USA, 105 (2008) 18355.

[27] Lin B., Yu J. and Rice S. A., Phys. Rev. E, 62 (2000) 3909. 